\title{
Obtención de tableros de fibras (HDF) a partir de papel periódico
}

\author{
Obtaining high density fiber boards from newsprint
}

\author{
Renzo Ceccarelli Lombardi ${ }^{1}$ y Hector E. Gonzales Mora ${ }^{2}$
}

\begin{abstract}
Resumen
En el presente trabajo se procedió a evaluar las propiedades físicas y mecánicas de tableros de fibra elaborados con fibras recicladas de papel periódico. Para la elaboración de los tableros se utilizó el método de la vía húmeda. Se caracterizó la materia prima (papel periódico) encontrándose una cantidad importante de lignina residual (25,69\%) que permitió la consolidación de los tableros. Según la evaluación de las propiedades físicas se observó que para las pruebas de incremento en masa y en volumen por absorción de agua, los tableros obtuvieron valores que los descalifica, sin embargo se debe resaltar que no se usó colofonia para mejorar esta propiedad. Con respecto a la densidad se obtuvieron valores que califican a los tableros como "tableros duros" y la blancura se vio afectada a medida que se aumentó la temperatura de consolidación. Dentro de la pruebas mecánicas se realizaron pruebas de dureza, alcanzando valores comparables al plástico duro (polipropileno virgen), además se realizaron pruebas de flexión tanto para el MOR y MOE, obteniendo resultados con menores rendimientos a los mencionados por otros autores, sin embargo los valores del presente trabajo están dentro del rango que los autores mencionan.
\end{abstract}

Palabras clave: tablero de fibras de alta densidad; papel periódico; propiedades físicas; propiedades mecánicas.

\begin{abstract}
The main objective of the study was to evaluate the physical and mechanical properties of fiber boards made from recycled fibers of newsprint. For the elaboration of boards the method of the humid route was used. The raw material (newsprint) had a significant amount of residual lignin $(25,69 \%)$ that allowed the consolidation of the boards. Regarding the evaluation of the physical properties on the mass and volume increment by water absorption test, the boards obtained values that disqualify them but it was due to the fact that colofonia was not used to improve this property, The density obtained values that describe to boards as "hard boards" and the whiteness were affected as the temperature of consolidation increased. In the mechanical tests hardness tests were performed, reaching comparable values as those of hard plastic (virgin polypropylene). In addition, MOR and MOE tests were performed, obtaining results with smaller yields to the ones mentioned by other authors; nevertheless the values of the present study are within the rank presented on similar tests.
\end{abstract}

Key words: HDF boards; newsprint; physical properties; mechanical properties.

${ }^{1}$ Minera Sulliden Shauindo SAC, Perú.

${ }^{2}$ Facultad de Ciencias Forestales, Universidad Nacional Agraria La Molina, Perú. E-mail: egonzales@lamolina.edu.pe 


\section{Introducción}

El Perú importa anualmente un promedio de 54700 toneladas de papel periódico al año (SUNAD) y se recicla menos del $20 \%$, transformándose en productos de baja calidad como en papel higiénico y cartón. El papel periódico difiere de otros papeles en que es hecho de pulpas de alto rendimiento, conservando sus componentes químicos, entre ellos la lignina, componente muy importante por su propiedad de termoplasticidad, característica que hace técnicamente viable la etapa de consolidación en la formación de tableros de fibra por medio de la vía húmeda.

El valor de la producción mundial de tableros, para el año 2002 fue de 182,1 millones de $\mathrm{m}^{3}$, siendo el principal productor del mundo los Estados Unidos con 40,6 millones de $\mathrm{m}^{3}$; el segundo productor mundial es China con 19,5 millones de $\mathrm{m}^{3}$ (Agrocadenas 2003).

La producción mundial de tableros de madera creció a una tasa de $3 \%$ entre 1998 y el año 2002. Por países, pese a la marcada disminución del mayor productor, Estados Unidos que decreció al 2,3\% anual promedio, los restantes productores, China, Canadá, Alemania, Brasil, Francia y Rusia crecieron a tasas de $15 \%, 5 \%$, $3 \%, 11 \%, 3,4 \%$ y $13 \%$ respectivamente. La tendencia del líder fue seguida solamente por Indonesia, que disminuyó su producción al pasar de $10163000 \mathrm{~m}^{3}$ en 1997 a $8118000 \mathrm{~m}^{3}$ en 2002 (Agrocadenas 2003).

Según (Kollman 1975) el mejor indicador para clasificar los tableros de fibra es la densidad; el autor menciona que los tableros de fibras comúnmente se encuentran en el rango de 0,02 a $1,45 \mathrm{~g} / \mathrm{cm}^{3}$ de densidad. Los tableros de fibra se clasifican según la vía de formación y los divide en tres grandes grupos según su vía de fabricación (vía humeda, vía seca y vía semiseca) (FAO 1975).

La pulpa mecánica de piedra, utilizada para la elaboración de papel periódico está formada por fibras individuales, fibras rotas, finos y haces de fibras burdas ya que la pulpa no se hidrata en el batido, sino que tiende a quebrarse, pro- duciendo así papeles voluminosos. Además, los constituyentes fibrosos de la pulpa mecánica se pueden clasificar como largos, de 0,8 a 4,5 $\mathrm{mm}$; cortos, de 0,2 a $0,8 \mathrm{~mm}$; y finos, que por su parte se clasifican como harina, de 0,02 a 0,2 $\mathrm{mm}$ y mucílagos con menos de 0,02 mm). La proporción de estos componentes determina las propiedades de espesamiento de la pulpa, la resistencia en húmedo y la resistencia en seco del papel. La cantidad de finos, elementos retenidos en tamiz de 150 mesh en las pulpas mecánicas varia habitualmente del $40 \%$ al 60 \% de la pulpa (Casey 1990).

La cantidad de pulpa mecánica utilizada en el papel periódico y en el destinado al empapelado es de $70 \%$ a $100 \%$, pero puede ser reforzado con una mezcla de hasta el 30\% de pulpa química para aportar fibras largas y mejorar las propiedades del papel periódico (Casey 1990).

Aunque en el Perú no se fabrica pulpa mecánica para el papel periódico, existen volúmenes importantes de importación, registrados por la Superintendencia Nacional de Aduanas, quienes en su reglamento técnico definen las características técnicas del papel periódico. En el arancel de aduanas se señala en el capítulo 48, nota 3 , que se considera al papel prensa o periódico como el papel sin estucar ni recubrir del tipo del utilizado para la impresión de diarios, en el que por lo menos el $65 \%$ en masa del contenido total fibroso esté constituido por fibras de madera obtenidas por procedimiento mecánico o químico mecánico, sin encolar o muy ligeramente encolado, cuyo índice de rugosidad, medido en el aparato Parker Print Surf (1 Mpa) sobre cada una de las caras, sea superior a 2,5 micras y de gramaje entre $40 \mathrm{~g} / \mathrm{m}^{2}$ y $65 \mathrm{~g} / \mathrm{m}^{2}$, y una blancura no mayor a $60 \%$. (ENA 1998). El valor de rugosidad se interpreta como la variación de espesor, con respecto a la línea base de una de sus caras.

Suchsland (1998) realizó experimentos en la Universidad del Estado de Michigan, con el fin de comparar propiedades sobre dos métodos de formación de tableros de fibras (vía húmeda y vía seca) con diferentes tipos de material. Los resultados alcanzados fueron prometedores, los 
tableros de fibras elaborados a base del reciclaje de papel periódico (vía húmeda), mostraron un valor MOR muy respetable, asimismo ya que no se tenía información de estudios sobre el reuso de la fibra del papel periódico, resalta la importancia de estos datos como también el carácter innovador del estudio. Además, sugiere que a una alta presión y una alta temperatura de prensa, debe ocurrir una adherencia de lignina importante.

En el presente trabajo se evaluó el comportamiento de tableros de fibra elaborados en base a papel periódico reciclado, en función a tres variables de producción: temperatura, presión y tiempo.

\section{Materiales y Métodos}

\section{Lugar de ejecución}

El presente trabajo de investigación se ejecutó en los Laboratorios del Área de Transformación Química y de Ensayos Tecnológicos de la Madera, departamento de Industrias Forestales de la Universidad Nacional Agraria La Molina (Lima, Perú) y en el Laboratorio de Control de Calidad de la empresa Fibraforte (Lima, Perú).

\section{Materiales y Equipos}

\section{Materia Prima}

Para el presente estudio se empleó papel periódico obtenido de muestras de ejemplares de todos los diarios informativos escritos que circulan en la ciudad de Lima. Para el cálculo de la cantidad en masa de la materia prima, se tomó la masa promedio de cada uno de los ejemplares una semana, así como también los respectivos tirajes, para luego dar una ponderación y mezclar el papel periódico en las proporciones correspondientes según la cantidad emitida, como masa, de cada diario.

A fin de verificar la presencia de pulpa mecánica en los diarios, se hizo una evaluación de la presencia de lignina en los diarios, indicador de pulpa mecánica, empleando la prueba cualitativa de coloración con floroglucinol.

\section{Equipos}

Se emplearon los siguientes equipos de laboratorio:

- Horno de secado a $105^{\circ} \mathrm{C}$

- Balanza analítica Ohaus, de 220v, capacidad de $200 \mathrm{~g}$, con una precisión de $0,1 \mathrm{mg}$

- Balanza portátil standard Ohaus LS 200, 12v, capacidad de $200 \mathrm{~g}$ con una precisión de $0,1 \mathrm{~g}$

- Formadora de hojas de laboratorio

- Equipo para medir drenabilidad de pulpa Schopper-Riegler

- Clasificador de fibras Bauer Mc-Nett

- Prensa de platos fríos de 30 x $30 \mathrm{~cm}$

- Bomba de vacío

- Campana desecadora con silicagel

- Mufla Thermolyne 48000, de 220 v, temperatura máxima de $1200{ }^{\circ} \mathrm{C}$

- Agitador magnético

Materiales y reactivos químicos

Los materiales empleados en el presente estudio fueron: probetas graduadas de 50, 100, 250, 500 y $1000 \mathrm{ml}$; pipetas de 5, 15 y $25 \mathrm{ml}$; vasos pirex de 50, 100 y $250 \mathrm{ml}$; cronómetro de precisión; baguetas de vidrio; pinzas; termómetro y papel secante.

Los reactivos químicos utilizados en el presente estudio:

- Ácido clorhídrico 4N

- Yoduro de potasio $1 \mathrm{M}$

- Tiosulfato de sodio $0,1 \mathrm{~N}$

- Hipoclorito de sodio 10\%

- Floroglucinol

- Solución de almidón $0,1 \%$

\section{Metodología}

Con los pesos promedios y los tirajes de cada ejemplar (diarios informativos que circulan en la ciudad de Lima) se procedió al rasgado manual en las proporciones adecuadas (en masa). Luego, se dejó 48 horas a temperatura ambiente para poder efectuar la preparación de la materia prima. 
Preparada la mezcla de papel periódico rasgado manualmente, se procedió a embolsar herméticamente muestras de $75 \mathrm{~g}$ de papel periódico (masa seca) por cada una para evitar el intercambio de humedad. Al final se preparó 90 muestras para la elaboración de los tableros de fibra, 81 para los ensayos y 9 para pruebas preliminares.

Los ensayos se realizaron en tres etapas:

\section{Evaluación de la materia prima}

En esta etapa se evaluó las características químicas de la materia prima.

Para la determinación del contenido de celulosa, expresado en porcentaje, se empleó el método Kurschner y Hoffner. La lignina, expresada en porcentaje, se determinó bajo las Normas TAPPI T222 om-88. Los extractivos, expresados en porcentaje, fueron determinados bajo la Norma TAPPI T6 os-59. Para la determinación del contenido de cenizas en la materia prima, expresado en porcentaje, se empleó la Norma ASTM D-1762. El contenido de sílice de la materia prima fue determinado por el método del ácido clorhídrico, norma según Laboratorio de Pulpa y Papel de la UNALM.

Se determinó el contenido de hemicelulosa expresado en porcentaje:

$$
\operatorname{Hem}(\%)=100-(\text { Extr }+\mathrm{Cel}+\mathrm{lig}+\mathrm{Cz})
$$

Donde:

Hem $=$ Cantidad de Hemicelulosa, expresada en porcentaje

Extr $=$ Contenido de Extractivos, expresado en porcentaje

Lig = Contenido de lignina, expresado en porcentaje

$\mathrm{Cel}=$ Contenido de celulosa, expresado en porcentaje

$\mathrm{Cz}=$ contenido de ceniza, expresado en porcentaje

Para la determinación del contenido de humedad en la materia prima, expresado en porcentaje, se empleó la Norma ASTM D-1762. La clasificación de fibras siguió la Norma TAPPI $233 \mathrm{~cm}-95$. La longitud de las fibras se determinó de acuerdo a norma interna del Laboratorio de Pulpa y Papel de la UNALM.

\section{Preparación de tableros de fibra}

\section{- Elaboración del manto fibroso (MAT)}

Se tomó $75 \mathrm{~g}$ de papel periódico (masa seca) y se colocó la misma dentro de un balde pequeño, luego se enrasó a un volumen de 2 litros con agua y se transfirió al desintegrador. Se desintegró a 75000 vueltas equivalente a 10 minutos de desintegración. Se llevó el volumen desintegrado a un balde mediano y se agregó 1,75 litros de agua: total 75 gramos de pulpa en $3750 \mathrm{ml}$, equivalente a una consistencia de $2 \%$ de pulpa. Por otro lado se conectó el embudo Buchner, con la malla $\mathrm{N}^{\circ} 150$, a un kitasato de 4 litros y que a su vez estuvo conectado con la bomba de vacío. Se vertió la pulpa, en dispersión acuosa, al embudo Buchner en forma continua tratando de no formar capas o estratos. Se retiró el manto fibroso formado (MAT) tratando de conservar su forma original con ayuda de una espátula; luego se determinó la masa del MAT $\left(\mathrm{P}_{1}\right)$.

\section{- Prensado en frio}

Se colocó 2 filtros de tela sobre el tablero de la prensa y sobre esta se colocó el MAT y sobre él un disco metálico. Se llevó todo el conjunto a la prensa y se aplico una presión específica de $4,2 \mathrm{~kg} / \mathrm{cm}^{2}$ por un periodo de 2 minutos. Se retiró el MAT de la prensa, se separo además los fieltros de tela y el disco metálico. Al final de esta etapa se obtuvo un manto fibroso de menor espesor al que se le ha eliminado una gran cantidad de agua libre; se determinó la masa del MAT $\left(\mathrm{P}_{2}\right)$.

\section{- Prensado en caliente}

Se controló la temperatura de trabajo de los platos calientes, para esta etapa $180^{\circ} \mathrm{C} \pm 5^{\circ} \mathrm{C}$. Sobre el MAT, prensado en frío, se colocó el "Mailen" (plástico termoestable) y en la otra cara del MAT se ubicó la malla metálica. Se ubicó el conjunto entre los platos calientes y se procedió a elevar el pistón de la prensa (se debe elevar la presión con mucho cuidado debido a la evacuación rápida del vapor). Se aplicó el esquema de Presión, temperatura y tiempo mostrada en la figura $\mathrm{N}^{\circ} 06$. Luego se procedió a un acondicionamiento del tablero a una tem- 
peratura y humedad relativa del medio por 30 minutos. Al final de esta etapa se determinó la masa del MAT $\left(\mathrm{P}_{3}\right)$ y se selló herméticamente el tablero en bolsas de plástico.

\section{Evaluación de las propiedades físicas y mecánicas de los tableros de fibra}

Se evaluaron las siguientes propiedades físicas: hinchamiento, tanto en incremento en peso como incremento en volumen, expresado en porcentaje, siguiendo la metodología de la Norma ISO r 768 (1968); la blancura, siguiendo la Norma ISO 3688; la densidad, expresada en $\mathrm{gr} / \mathrm{cm}^{3}$, fue determinada por la Norma Interna del Laboratorio de Pulpa y Papel de la UNALM.

Se evaluaron las siguientes propiedades mecánicas: flexión estática, según la norma ASTM D 1037 - 64 (1964), se efectuaron modificación en longitud con el fin de adaptar las muestras a la prensa.

La expresión de la prueba de flexión se hizo mediante los siguientes parámetros:

Módulo de elasticidad (MOE): expresado en $\mathrm{kg} / \mathrm{cm}^{2}$, cuya formula empleada se indica a continuación:

$$
M O E=\frac{P / L^{3}}{4 * b^{3} * d^{*} Y_{1}}
$$

Donde:

P: carga al límite proporcional $(\mathrm{kg})$

L: luz $(\mathrm{cm})$

b: espesor $(\mathrm{cm})$

$\mathrm{d}$ : ancho de la probeta $(\mathrm{cm})$

Y: deflexión al límite proporcional $(\mathrm{cm})$

Módulo de Ruptura (MOR): se expresa en $\mathrm{kg} / \mathrm{cm}^{2}$ y su formula se presenta a continuación:

$$
M O R=\frac{3 * P}{2 * b^{2} * d} L
$$

Donde:

P: carga máxima $(\mathrm{kg})$

L: luz $(\mathrm{cm})$

b: espesor $(\mathrm{cm})$

$\mathrm{d}$ : ancho de la probeta $(\mathrm{cm})$

$$
\mathrm{T}_{\mathrm{p}}=\frac{\mathrm{P}}{\mathrm{A}}
$$

Se efectuó la prueba de tracción perpendicular a las caras según la Norma ASTM D 1037 - 64 (1964). Los resultados del ensayo se expresa en $\mathrm{kg} / \mathrm{cm}^{2}$ y su fórmula se presenta a continuación:

Donde:

$\mathrm{T}_{\mathrm{p}}$ : tracción perpendicular $\left(\mathrm{kg} / \mathrm{cm}^{2}\right)$

P: carga $(\mathrm{kg})$

A: área del tablero $\left(\mathrm{cm}^{2}\right)$

Los ensayos de dureza se realizaron bajo la norma DIN 53505 equivalente a la Norma ISO 868. Se expresó en la escala Shore.

\section{Diseño estadístico}

El análisis para el trabajo se realizó con ayuda de los programas STATGRAPHICS Plus Versión 3.0 y STATISTICA Versión 5.0 con un diseño experimental $3^{3}$; es decir que estuvo comprendido por 3 niveles y 3 factores $(3 \times 3 \times 3)$ y tiene como modelo la siguiente ecuación:

$$
\begin{gathered}
Y=\beta_{0}+\beta_{1} \alpha_{1}+\beta_{2} \alpha_{2}+\beta_{3} \alpha_{3}+\beta_{4} \alpha_{1}^{2}+\beta_{5} \alpha_{1} \alpha_{2}+\beta_{6} \alpha_{1} \\
\alpha_{3}+\beta_{7} \alpha_{2}^{2}+\beta_{8} \alpha_{2} \alpha_{3}+\beta_{9} \alpha_{3}^{2}+\varepsilon
\end{gathered}
$$

Donde:

$\beta_{1} \ldots \beta_{9}$ : Coeficientes de regresión de cada variable o de sus interacciones.

$\alpha_{1} \ldots \alpha_{3}$ : Variables (presión, tiempo, temperatura)

$\alpha_{1} \alpha_{2}, \alpha_{2} \alpha_{3}, \alpha_{1} \alpha_{3}$ : interacción

$\varepsilon$ : efecto del error aleatorio 


\begin{tabular}{|c|c|c|c|c|c|c|c|c|c|}
\hline \multirow{3}{*}{ Tiempos } & \multicolumn{9}{|c|}{ Niveles de Presión y Temperatura } \\
\hline & \multicolumn{3}{|c|}{ Presión $1=3000$ lb } & \multicolumn{3}{|c|}{ Presión $2=4000 \mathrm{lb}$} & \multicolumn{3}{|c|}{ Presión $3=5000$ lb } \\
\hline & T1 & $\mathbf{T 2}$ & T3 & T1 & $\mathbf{T 2}$ & T3 & T1 & $\mathbf{T} 2$ & T3 \\
\hline $\mathrm{t} 1$ & $\mathrm{P}_{1} \mathrm{~T}_{1} \mathrm{t}_{1}$ & $\mathrm{P}_{1} \mathrm{~T}_{2} \mathrm{t}_{1}$ & $\mathrm{P}_{1} \mathrm{~T}_{3} \mathrm{t}_{1}$ & $\mathrm{P}_{2} \mathrm{~T}_{1} \mathrm{t}_{1}$ & $\mathrm{P}_{2} \mathrm{~T}_{2} \mathrm{t}_{1}$ & $\mathrm{P}_{2} \mathrm{~T}_{3} \mathrm{t}_{1}$ & $\mathrm{P}_{3} \mathrm{~T}_{1} \mathrm{t}_{1}$ & $\mathrm{P}_{3} \mathrm{~T}_{2} \mathrm{t}_{1}$ & $\mathrm{P}_{3} \mathrm{~T}_{3} \mathrm{t}_{1}$ \\
\hline $\mathrm{t} 1$ & $\mathrm{P}_{1} \mathrm{~T}_{1} \mathrm{t}_{1}$ & $\mathrm{P}_{1} \mathrm{~T}_{2} \mathrm{t}_{1}$ & $\mathrm{P}_{1} \mathrm{~T}_{3} \mathrm{t}_{1}$ & $\mathrm{P}_{2} \mathrm{~T}_{1} \mathrm{t}_{1}$ & $\mathrm{P}_{2} \mathrm{~T}_{2} \mathrm{t}_{1}$ & $\mathrm{P}_{2} \mathrm{~T}_{3} \mathrm{t}_{1}$ & $\mathrm{P}_{3} \mathrm{~T}_{1} \mathrm{t}_{1}$ & $\mathrm{P}_{3} \mathrm{~T}_{2} \mathrm{t}_{1}$ & $\mathrm{P}_{3} \mathrm{~T}_{3} \mathrm{t}_{1}$ \\
\hline $\mathrm{t} 1$ & $\mathrm{P}_{1} \mathrm{~T}_{1} \mathrm{t}_{1}$ & $\mathrm{P}_{1} \mathrm{~T}_{2} \mathrm{t}_{1}$ & $\mathrm{P}_{1} \mathrm{~T}_{3} \mathrm{t}_{1}$ & $\mathrm{P}_{2} \mathrm{~T}_{1} \mathrm{t}_{1}$ & $\mathrm{P}_{2} \mathrm{~T}_{2} \mathrm{t}_{1}$ & $\mathrm{P}_{2} \mathrm{~T}_{3} \mathrm{t}_{1}$ & $\mathrm{P}_{3} \mathrm{~T}_{1} \mathrm{t}_{1}$ & $\mathrm{P}_{3} \mathrm{~T}_{2} \mathrm{t}_{1}$ & $\mathrm{P}_{3} \mathrm{~T}_{3} \mathrm{t}_{1}$ \\
\hline $\mathrm{t} 2$ & $\mathrm{P}_{1} \mathrm{~T}_{1} \mathrm{t}_{2}$ & $\mathrm{P}_{1} \mathrm{~T}_{2} \mathrm{t}_{2}$ & $\mathrm{P}_{1} \mathrm{~T}_{3} \mathrm{t}_{2}$ & $\mathrm{P}_{2} \mathrm{~T}_{1} \mathrm{t}_{2}$ & $\mathrm{P}_{2} \mathrm{~T}_{2} \mathrm{t}_{2}$ & $\mathrm{P}_{2} \mathrm{~T}_{3} \mathrm{t}_{2}$ & $\mathrm{P}_{3} \mathrm{~T}_{1} \mathrm{t}_{2}$ & $\mathrm{P}_{3} \mathrm{~T}_{2} \mathrm{t}_{2}$ & $\mathrm{P}_{3} \mathrm{~T}_{3} \mathrm{t}_{2}$ \\
\hline $\mathrm{t} 2$ & $\mathrm{P}_{1} \mathrm{~T}_{1} \mathrm{t}_{2}$ & $\mathrm{P}_{1} \mathrm{~T}_{2} \mathrm{t}_{2}$ & $\mathrm{P}_{1} \mathrm{~T}_{3} \mathrm{t}_{2}$ & $\mathrm{P}_{2} \mathrm{~T}_{1} \mathrm{t}_{2}$ & $\mathrm{P}_{2} \mathrm{~T}_{2} \mathrm{t}_{2}$ & $\mathrm{P}_{2} \mathrm{~T}_{3} \mathrm{t}_{2}$ & $\mathrm{P}_{3} \mathrm{~T}_{1} \mathrm{t}_{2}$ & $\mathrm{P}_{3} \mathrm{~T}_{2} \mathrm{t}_{2}$ & $\mathrm{P}_{3} \mathrm{~T}_{3} \mathrm{t}_{2}$ \\
\hline $\mathrm{t} 2$ & $\mathrm{P}_{1} \mathrm{~T}_{1} \mathrm{t}_{2}$ & $\mathrm{P}_{1} \mathrm{~T}_{2} \mathrm{t}_{2}$ & $\mathrm{P}_{1} \mathrm{~T}_{3} \mathrm{t}_{2}$ & $\mathrm{P}_{2} \mathrm{~T}_{1} \mathrm{t}_{2}$ & $\mathrm{P}_{2} \mathrm{~T}_{2} \mathrm{t}_{2}$ & $\mathrm{P}_{2} \mathrm{~T}_{3} \mathrm{t}_{2}$ & $\mathrm{P}_{3} \mathrm{~T}_{1} \mathrm{t}_{2}$ & $\mathrm{P}_{3} \mathrm{~T}_{2} \mathrm{t}_{2}$ & $\mathrm{P}_{3} \mathrm{~T}_{3} \mathrm{t}_{2}$ \\
\hline $\mathrm{t} 3$ & $\mathrm{P}_{1} \mathrm{~T}_{1} \mathrm{t}_{3}$ & $\mathrm{P}_{1} \mathrm{~T}_{2} \mathrm{t}_{3}$ & $\mathrm{P}_{1} \mathrm{~T}_{3} \mathrm{t}_{3}$ & $\mathrm{P}_{2} \mathrm{~T}_{1} \mathrm{t}_{3}$ & $\mathrm{P}_{2} \mathrm{~T}_{2} \mathrm{t}_{3}$ & $\mathrm{P}_{2} \mathrm{~T}_{3} \mathrm{t}_{3}$ & $\mathrm{P}_{3} \mathrm{~T}_{1} \mathrm{t}_{3}$ & $\mathrm{P}_{3} \mathrm{~T}_{2} \mathrm{t}_{3}$ & $\mathrm{P}_{3} \mathrm{~T}_{3} \mathrm{t}_{3}$ \\
\hline $\mathrm{t} 3$ & $\mathrm{P}_{1} \mathrm{~T}_{1} \mathrm{t}_{3}$ & $\mathrm{P}_{1} \mathrm{~T}_{2} \mathrm{t}_{3}$ & $\mathrm{P}_{1} \mathrm{~T}_{3} \mathrm{t}_{3}$ & $\mathrm{P}_{2} \mathrm{~T}_{1} \mathrm{t}_{3}$ & $\mathrm{P}_{2} \mathrm{~T}_{2} \mathrm{t}_{3}$ & $\mathrm{P}_{2} \mathrm{~T}_{3} \mathrm{t}_{3}$ & $\mathrm{P}_{3} \mathrm{~T}_{1} \mathrm{t}_{3}$ & $\mathrm{P}_{3} \mathrm{~T}_{2} \mathrm{t}_{3}$ & $\mathrm{P}_{3} \mathrm{~T}_{3} \mathrm{t}_{3}$ \\
\hline $\mathrm{t} 3$ & $\mathrm{P}_{1} \mathrm{~T}_{1} \mathrm{t}_{3}$ & $\mathrm{P}_{1} \mathrm{~T}_{2} \mathrm{t}_{3}$ & $\mathrm{P}_{1} \mathrm{~T}_{3} \mathrm{t}_{3}$ & $\mathrm{P}_{2} \mathrm{~T}_{1} \mathrm{t}_{3}$ & $\mathrm{P}_{2} \mathrm{~T}_{2} \mathrm{t}_{3}$ & $\mathrm{P}_{2} \mathrm{~T}_{3} \mathrm{t}_{3}$ & $\mathrm{P}_{3} \mathrm{~T}_{1} \mathrm{t}_{3}$ & $\mathrm{P}_{3} \mathrm{~T}_{2} \mathrm{t}_{3}$ & $\mathrm{P}_{3} \mathrm{~T}_{3} \mathrm{t}_{3}$ \\
\hline
\end{tabular}

Cuadro 1. Matriz de 3 niveles y 3 factores del diseño experimental.

En este estudio se utilizó este modelo que comprende 3 factores, a fin de evaluar la influencia de las variables independientes y de sus interacciones sobre los resultados propuestos. El Cuadro 1 muestra los tratamientos.

\section{Resultados}

\section{Evaluación química de la materia prima}

En el Cuadro 2 se puede observar los valores en porcentaje de los componentes químicos de la materia prima. Los análisis se realizaron con muestras cuyo contenido de humedad fue $12,90 \%$. El contenido de lignina fue de 25,69\% lo cual confirma que la materia prima fue tratada mecánicamente, también se realizó pruebas cualitativas con reactivos (Floroglucinol) que producen un color rojo - violeta al reaccionar con la lignina del papel periódico. La presencia de lignina es muy importante ya que por su termoplasticidad favorece en la consolidación de los tableros de fibra.

\section{Evaluación de las fibras}

Se realizó la clasificación de las fibras y se sometieron dos muestras de 10 gramos cada una. Los resultados obtenidos se presentan en el Cuadro 3.

\section{Evaluaciones físico-mecánicas}

\section{Prueba de blancura}

Según el análisis estadístico y el análisis de variancia respectivo, se encontró una influencia, con un nivel altamente significativo, de los factores presión y temperatura sobre los valores de la blancura de los tableros de fibra. En la Figura 1 son representadas las variaciones de los valores de la blancura y de los dos factores mencionados.

Se observa que los valores de blancura disminuyen conforme aumentan los valores de la temperatura y la presión, siendo esta última la variable que menos influye en esta relación. El indicador de este análisis está dado por el nivel de significancia y de manera práctica, observando la pendiente de la variable. A mayor temperatura se produce una disminución en la blancura de las fibras, mientras que la presión actúa, de manera simultánea con la temperatura. Asimismo, la temperatura y la presión pueden modificar las partículas de tinta aún remanentes en las fibras, desfavoreciendo los valores de blancura del tablero.

\section{Densidad}

Los valores de la densidad de los tableros estuvieron en un rango de 0,81 a $0,91 \mathrm{gr} / \mathrm{cm}^{3}$, 


\begin{tabular}{|c|c|}
\hline Componente químico & Porcentaje (\%) \\
\hline Celulosa & 67,045 \\
\hline Lignina & 25,69 \\
\hline Número Hypo & 10,74 \\
\hline Hemicelulosa & 2,2545 \\
\hline Extractivos & 4,355 \\
\hline Cenizas & 0,655 \\
\hline Sílice & 0,385 \\
\hline
\end{tabular}

Cuadro 2. Componentes químicos de la materia prima.

\begin{tabular}{|c|c|c|}
\hline & Cantidad (g) & $\%$ \\
\hline Filtro 30 & 3,5365 & 35,365 \\
\hline Filtro 50 & 2,6762 & 26,765 \\
\hline Filtro 100 & 0,6054 & 6,055 \\
\hline Filtro 200 & 1,3663 & 13,675 \\
\hline Diferencia & 1,8156 & 18,14 \\
\hline
\end{tabular}

Cuadro 3. Clasificación de fibras de la materia prima.

siendo los valores más altos los obtenidos con el tratamiento $\mathrm{P}_{1} \mathrm{~T}_{1} \mathrm{t}_{2}$, a una temperatura de 140 ${ }^{\circ} \mathrm{C}$, tiempo de consolidación de 5 minutos y a una presión manométrica de $15,5 \mathrm{~kg} / \mathrm{cm}^{2}$.

Del análisis de variancia, se deduce que los factores temperatura y tiempo no presentan influencia significativa sobre la variación de los valores de densidad de los tableros. Sin embargo, se percibe en la Figura 2 una variación de resultados por influencia de ambos factores, con una probabilidad del $99 \%$, siendo el factor tiempo el que, aparentemente menos influye en esta relación. En la Figura 2 se observa que a medida que la temperatura y el tiempo aumentan, los valores de la densidad disminuyen.

\section{Aumento en masa por absorción de agua}

El incremento en masa de los tableros estuvo en un rango entre los valores de $79 \%$ a $84 \%$, siendo el valor más bajo el obtenido con el tratamiento $\mathrm{P}_{2} \mathrm{~T}_{3} \mathrm{t}_{3}$, con una presión de $20,66 \mathrm{~kg} /$ $\mathrm{cm}^{2}$, temperatura de $160^{\circ} \mathrm{C}$ y un tiempo de consolidación de 7 minutos. Según el análisis de variancia, la influencia de los factores presión y temperatura, sobre los valores de aumento en masa de los tableros, es altamente

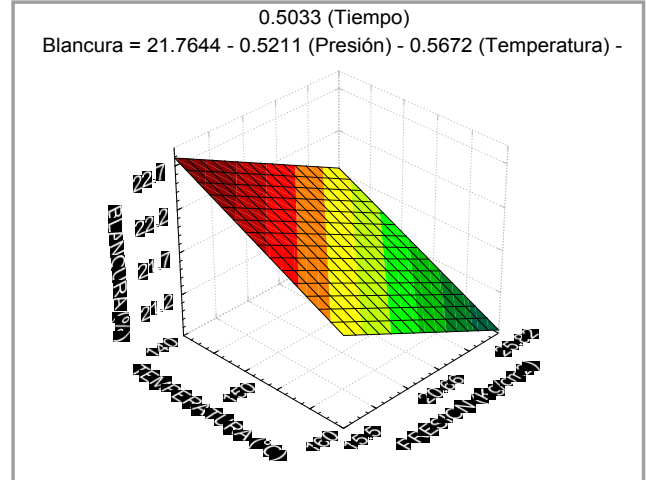

Figura 1. Superficie de respuesta de la influencia de la temperatura y la presión sobre los valores de blancura de los tableros del presente estudio.

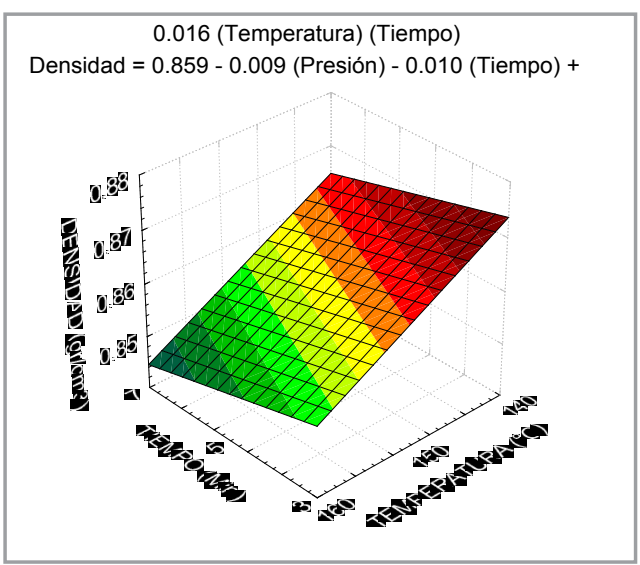

Figura 2. Superficie de respuesta de la influencia de la temperatura y el tiempo sobre los valores de densidad de los tableros del presente estudio.

significativa, siendo la temperatura el factor que menos influye en este ensayo.

La influencia de los factores se percibe de manera práctica al observar en la Figura 3 la pendiente: sobre la misma se muestra que a medida que aumenta la presión, los valores del aumento en masa de los tableros disminuye.

\section{Aumento en volumen por absorción de agua}

El aumento en volumen de los tableros estuvo en un rango entre los valores de $45 \%$ a $48 \%$ siendo el mayor valor el obtenido en el tratamiento $\mathrm{P}_{1} \mathrm{~T}_{3} \mathrm{t}_{3}$ a una presión de $15,5 \mathrm{~kg} / \mathrm{cm}^{2}$, una temperatura de $160^{\circ} \mathrm{C}$ y un tiempo de consolidación de 7 minutos. 


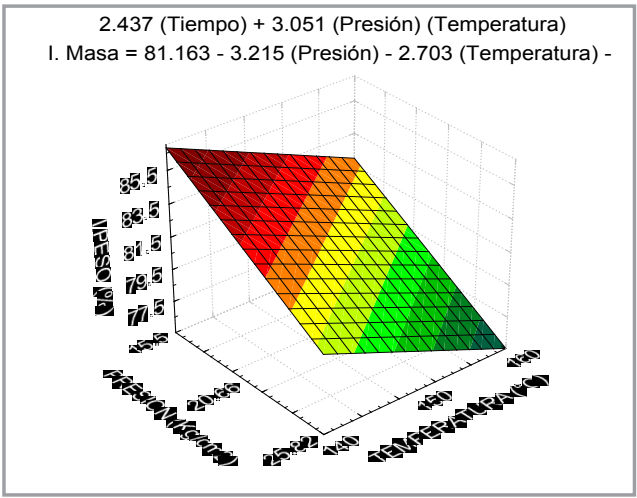

Figura 3. Superficie de respuesta de la influencia de la temperatura y la presión sobre los valores de aumento en masa por absorción de agua, de los tableros del presente estudio.

Del análisis de variancia entre los factores temperatura y presión para la prueba de aumento en volumen de los tableros, se encontró que ambos factores no presentaron una influencia significativa (95\%). Sin embargo, en la Figura 4 se observa una variación del aumento de masa afectado por el tiempo y temperatura, con una probabilidad del $90 \%$, siendo el tiempo el factor que menos influye en estos resultados.

\section{Dureza}

Los valores de la dureza de los tableros se obtuvieron dentro de un rango de 11 a 19 Shore D, siendo los valores más altos los obtenidos con el tratamiento $\mathrm{P}_{2} \mathrm{~T}_{2} \mathrm{t}_{3}$, a una presión manométrica $20,66 \mathrm{~kg} / \mathrm{cm}^{2}$, a una temperatura de $150^{\circ} \mathrm{C}$ y un tiempo de consolidación de 7 minutos.

Del análisis de variancia, se deduce que los factores temperatura y tiempo no presentan influencia significativa sobre la variación de los valores de dureza de los tableros. Sin embargo, se percibe en la Figura 5 una variación de resultados por influencia de ambos factores, con una probabilidad del $85 \%$, siendo el factor temperatura el que más influye en esta relación.

En la Figura 5 se observa que a medida que aumenta la temperatura, los valores de la dureza también incrementan. La elevación de la temperatura favorece la plastificación de la

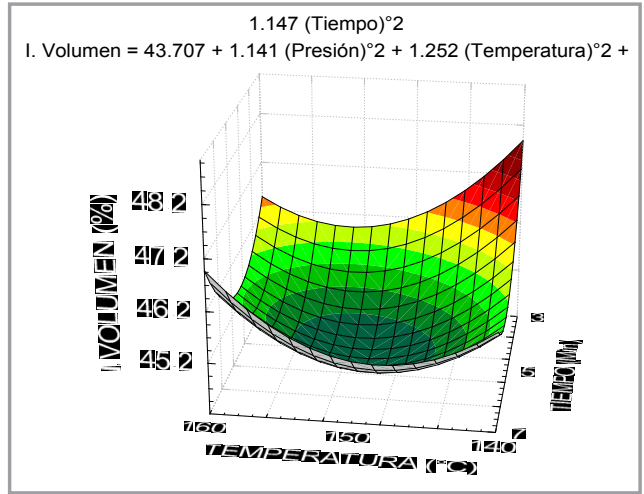

Figura 4. Superficie de respuesta de la influencia de la temperatura y tiempo sobre los valores de aumento de volumen por absorción de agua de los tableros del presente estudio.

lignina remanente de las fibras secundarias utilizadas para elaborar los tableros. La fibra secundaria empleada presenta una cantidad importante de lignina, verificado por el número Hypo, cuyos valores se encuentran en relación directa con la lignina.

\section{Tracción}

Los valores de tracción de los tableros se obtuvieron dentro de un rango de 2,0 a $5,0 \mathrm{~kg} /$ $\mathrm{cm}^{2}$, siendo los valores más altos los obtenidos con el tratamiento $\mathrm{P}_{1} \mathrm{~T}_{3} \mathrm{t}_{1}$, a una presión manométrica de $15,5 \mathrm{~kg} / \mathrm{cm}^{2}$, a una temperatura de $160^{\circ} \mathrm{C}$ y tiempo de consolidación de 7 minutos.

En la Figura 6 son representadas las variaciones de los valores de la tracción y de los dos factores mencionados. Se puede observar en la figura que a medida que aumenta la presión, los valores de tracción disminuyen, sin embargo el factor temperatura afecta positivamente a los valores de la tracción.

Según el análisis estadístico y el análisis de variancia respectivo se encontró una influencia, con un nivel altamente significativo, de los factores presión y temperatura sobre los valores de la tracción de los tableros de fibra.

\section{MOR}

Los valores de MOR de los tableros estuvieron dentro del rango de 300 a $450 \mathrm{~kg} / \mathrm{cm}^{2}$, 
Dureza $=15.8526+0.5006($ Temperatura $)-0.6117($ Temperatura $)($ Tiempo $)$

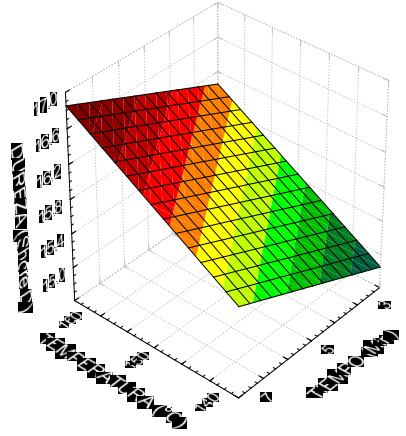

Figura 5. Superficie de respuesta de la influencia de la temperatura y tiempo sobre los valores de dureza de los tableros del presente estudio.

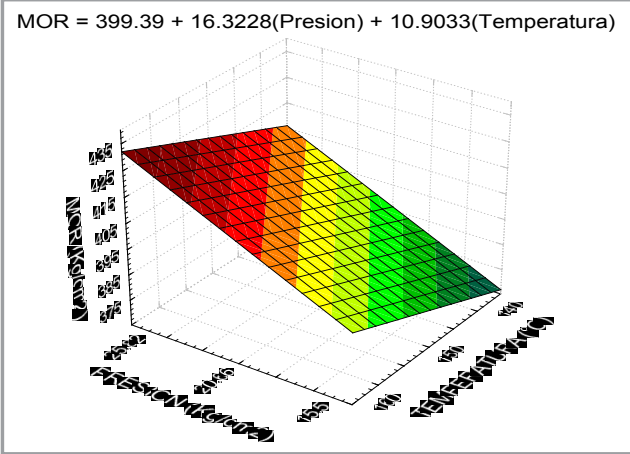

Figura 7. Superficie de respuesta de la influencia de la presión y de la temperatura sobre los valores de MOR de los tableros del presente estudio.

siendo los valores más altos los obtenidos con el tratamiento $\mathrm{P}_{2} \mathrm{~T}_{3} \mathrm{t}_{2}$, a una presión manométrica de $20,66 \mathrm{~kg} / \mathrm{cm}^{2}$, a una temperatura de $160^{\circ} \mathrm{C}$ y tiempo de consolidación de 5 minutos.

Según el análisis estadístico y el análisis de variancia respectivo, se encontró una influencia significativa del factor presión mientras que la temperatura muestra una probabilidad de ocurrencia de $85 \%$ sobre los valores de MOR de los tableros de fibra.

En la Figura 7 se puede observar que los valores de MOR se ven favorecidos a medida que aumenta la presión y la temperatura. La compactación sobre el manto fibroso y la temperatura que actúa sobre la plastificación de la lignina contribuyen a obtener mejores valores de MOR de tableros de fibra.

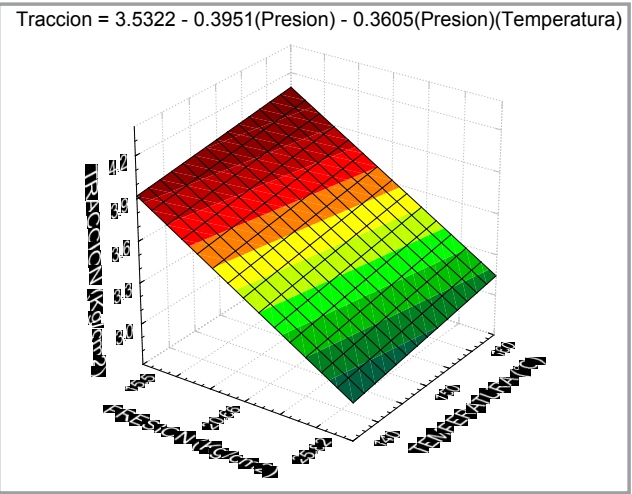

Figura 6. Superficie de respuesta de la influencia de la presión y de la temperatura sobre los valores de tracción de los tableros del presente estudio.

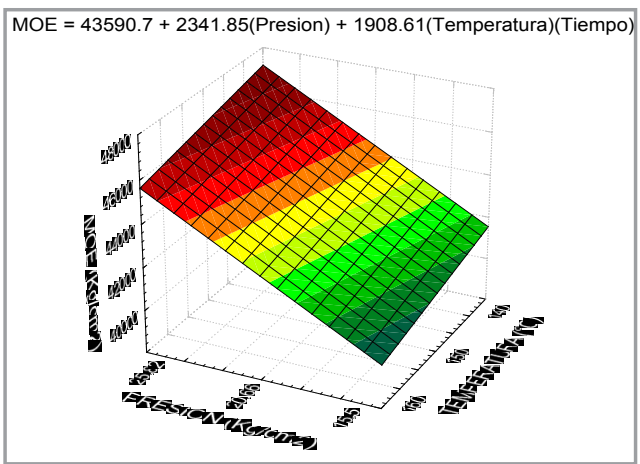

Figura 8. Superficie de respuesta de la influencia de la presión y de la temperatura sobre los valores de MOE de los tableros del presente estudio.

\section{MOE}

Los valores de MOE de los tableros se obtuvieron dentro de un rango de 30000 a 50000 $\mathrm{kg} / \mathrm{cm}^{2}$, siendo los valores más altos los obtenidos con el tratamiento $\mathrm{P}_{3} \mathrm{~T}_{1} \mathrm{t}_{2}$, a una presión manométrica de $25,82 \mathrm{~kg} / \mathrm{cm}^{2}$, a una temperatura de $140^{\circ} \mathrm{C}$ y tiempo de consolidación de 5 minutos.

Según el análisis estadístico y el análisis de variancia respectivo, se encontró una influencia significativa del factor presión mientras que la combinación entre los factores temperatura y tiempo muestra una probabilidad de ocurrencia de $85 \%$ sobre los valores de MOE de los tableros de fibra.

En la Figura 8 se puede observar que los va- 
lores de MOE se ven favorecidos a medida que aumenta la presión debido a la compactación sobre el manto fibroso disminuyendo los espacios vacíos entre las fibras dándole una mejor resistencia a los esfuerzos perpendiculares, sin embargo la temperatura actúa de forma inversa con respecto a los valores del MOE, a mayores temperaturas, la lignina residual de la materia prima hace los tableros mas duros, esta condición hace disminuir los valores del MOE.

\section{Discusión}

En las pulpas mecánicas se encuentran fibras largas $(0,8$ a $4,5 \mathrm{~mm})$, fibras cortas $(0,2$ a 0,8 $\mathrm{mm}$ ) y fibras muy finas que por su parte se clasifican como harinas $(0,02$ a $0,2 \mathrm{~mm})$ y mucílagos (menos de $0,02 \mathrm{~mm}$ ), y que la cantidad de fibras muy finas en la pulpa mecánica (la proporción de pulpa que pasa por un filtro de 100 ) incluye habitualmente del $30 \%$ al $60 \%$ de la pulpa según Casey (1990). En el Cuadro 14 se observa que la proporción que pasó el filtro 100 es de $37,87 \%$, este resultado concuerda con lo descrito anteriormente por el autor.

Casey (1990) menciona que los iones metálicos y los compuestos de los extractivos, inducidos térmicamente contribuyen a la reversión de color de las fibras de la pulpa mecánica. En las fibras secundarias hay una pequeña fracción de extractivos (4\%), aparentemente extractivos naturales remanentes, las tintas, por su composición química grasa, actúan como tales, por consiguiente contribuye a la reversión de color del tablero.

La elevación de la temperatura favorece la plastificación de la lignina remanente de las fibras secundarias utilizadas para elaborar los tableros. La fibra secundaria empleada presenta una cantidad importante de lignina, verificado por el número Hypo, cuyos valores se encuentran en relación directa con la lignina. Sin embargo, un exceso de temperatura afecta la estructura de la fibra, reduciendo su capacidad de unión y por consiguiente los valores de densidad del tablero. El alto porcentaje de fibras muy finas no permite el entrecruzamiento entre las fibras, reduciéndose la unión entre las fibras, afectando los valores de densidad. En la ecuación obtenida de densidad, el factor temperatura interactúa con el tiempo para que disminuya la densidad del tablero.

Kollman (1975) menciona una clasificación de tableros de fibra según la densidad, designados como tableros "duros" aquellos que tienen una densidad de 0,80 a $1,00 \mathrm{gr} / \mathrm{cm}^{3}$. Los tableros elaborados en el presente estudio se encuentran dentro de la clasificación de tablero duro. De la misma manera, en base a la clasificación de la FAO (1968), para los tableros de fibra, según la norma técnica ISO $\mathrm{N}^{\circ} 180$ / R818-1968, los tableros obtenidos se clasifican como tableros de densidad alta, por sus valores de densidad mayores a $0,80 \mathrm{~g} / \mathrm{cm}^{3}$.

Esta influencia de la presión durante la absorción puede ser explicada por el tamaño y cantidad de espacios libres que quedan dentro del tablero como consecuencia de una mayor presión de aplicación. Al reducirse la porosidad del tablero, disminuye la capacidad para almacenar agua entre las fibras del tablero. Asimismo, una presión y temperatura eleva$\mathrm{da}$, los espacios libres son menores y de menor tamaño, por lo que dificulta el ingreso del agua hacia el tablero.

Karikal (1996) reporta en sus ensayos porcentajes de $20 \%$ en aumento en masa del tablero, por absorción de agua. Asimismo, Kollman (1975) reporta resultados similares, entre 10\% a 30\% de absorción. Estos resultados están por debajo de los resultados obtenidos en el presente estudio, esto puede deberse a que en la elaboración de los tableros no se utilizó ni un tipo de aditivo hidrófugo, como la colofonia, que ayudan a disminuir la absorción de agua por parte de los tableros, haciéndolos más estables en medios húmedos.

El aumento en el volumen de los tableros ocurre porque en la pared celular de la fibras, específicamente en la capa S2 existen radicales hoxidrílicos los que son muy afines a compuestos polares como el agua. Por consiguiente, las fibras, al estar en un medio saturado, sus enlaces captan agua con facilidad (puente de hidrógeno), provocando un hinchamiento en 
las micro fibrillas y así el cambio dimensional. Este comportamiento se ve más favorecido en las fibras recicladas ya que la pared secundaria está muy fracturada, debido a que ha pasado por dos procesos de pulpeo, incrementándose la proporción de fibras muy finas.

En ensayos realizados por Kollman (1975) se obtuvieron valores de aumento en volumen de $14 \%$ a 25\%; asimismo, la FAO (1975) reportó valores entre $10 \%$ a $30 \%$. Estos resultados están muy por debajo de los obtenidos en el presente estudio; esta diferencia se debe a que en el presente estudio no se usó ningún aditivo para retardar el ingreso del agua, tal como la colofonia que minimiza los efectos de la humedad dentro del tablero.

La cantidad de lignina encontrada favorece en forma directa a la dureza de los tableros, obteniéndose tableros más duros a mayores temperaturas. Los valores de dureza obtenidos son muy similares a los valores de dureza del polipropileno virgen, material plástico utilizado como materia prima para la elaboración de calaminas plásticas (fábrica Fibraforte).

Los valores de la tracción se ven afectados por dos razones, la primera razón es por la cantidad de fibras finas que están presentes en la materia prima, disminuyendo así la capacidad de entrecruzamiento entre las fibras. La segunda razón recae en el efecto de la presión sobre las fibras, provocando la rotura de las fibras a medida que este factor aumenta, disminuyendo la capacidad de entrecruzamiento de las fibras.

Los valores obtenidos de MOR se encuentran dentro del rango reportado por Kollman (1975) que están entre 300 a $550 \mathrm{~kg} / \mathrm{cm}^{2}$, sin embargo los resultados del presente estudio son ligeramente menores a los reportados ya que solo se llegó hasta $450 \mathrm{~kg} / \mathrm{cm}^{2}$. Asimismo, los valores obtenidos de MOE se encuentran dentro del rango de valores reportado por la FAO (1968), reportando valores de 28000 a $56000 \mathrm{~kg} / \mathrm{cm}^{2}$, sin embargo los valores obtenidos no llegan a alcanzar el límite superior de los valores reportados por la FAO (1968).

\section{Conclusiones}

En la materia prima se encontró cantidades importantes de lignina residual (25,69\%), componente importante para la consolidación de los tableros de fibra.

La blancura de los tableros se ve afectada por la temperatura de consolidación, ya que esta provoca la reversión de color tanto en los extractivos remanentes de la madera y de las tintas de impresión presentes en la materia prima.

Los tableros de fibra obtenidos son muy permeables al agua, ya que los valores obtenidos en el incremento en masa y volumen por absorción de agua (79 a 84\%) y (45 a 48\%) respectivamente son muy elevados. Estos valores se encuentran muy por encima de los valores de las especificaciones técnicas de los tableros comerciales.

Con respecto a la dureza de los tableros, se puede decir que los valores son comparables con otros materiales como el polipropileno virgen (plástico).

Los valores de MOR de los tableros obtenidos (300 a $450 \mathrm{~kg} / \mathrm{cm}^{2}$ ) están dentro del rango de los valores de los tableros comerciales (300 a $550 \mathrm{~kg} / \mathrm{cm}^{2}$ ), sin embargo los tableros comerciales tienen un mejor rendimiento en esta propiedad mecánica.

Los valores de MOE de los tableros obtenidos $\left(30000\right.$ a $\left.50000 \mathrm{~kg} / \mathrm{cm}^{2}\right)$ están dentro del rango de los valores de los tableros comerciales (28000 a $56000 \mathrm{~kg} / \mathrm{cm}^{2}$ ), sin embargo los tableros comerciales poseen un mejor rendimiento en esta propiedad mecánica.

\section{Bibliografía}

ASTM. 1980. American Society for Testing Materials. Standard Wood Specifications Philadelphia.

Casey, J. 1990. Pulpa y Papel. Química y Tecnología Química. Vol I, II y III. Limusa MÉXICO. 
ENA (Escuela Nacional de Aduanas). 1997. Arancel de Aduanas, DS - N 119 - 97- EF.

FAO (Organización para la Alimentación y la Agricultura). 1968. Tableros Contrachapados y Otros Paneles a base de Madera. Roma, Italia. $248 \mathrm{p}$.

FAO (Organización para la Alimentación y la Agricultura). 1975. Consulta Mundial sobre Paneles a base de Madera. New York, USA.

ITINTEC. 1976. Norma 12:02 - 001, glosario de términos para la Industria de Pulpa, Papel y Cartón. Lima - Perú.

Kollman, F. 1975. Principles of Wood Science and Technology II. New York, USA.

López, M. 1998. Introducción a la Tecnología de Pulpa y Papel. Lima - Perú.

Moscoso, J. 1995. El maderero. Boletín Informativo $\mathrm{N}^{\circ} 13$. Redinfor - Perú.

Myers, G. 1987. Characterization of Fiberboard Pulp. Forest Products Journal 2 (30). 36 p.

Suchsland, O. 1998. Effect of Hardboard Process Variables on Fiberbonding. Forest Products Journal 33(4): $58-64$.

Wayne, W. 1993. Bioestadística, 3ra. Edición, Limusa. 667 p.

Yongsung, O. 1997. Fillers for Plywood Adhesives Made from acid Hydrolysis Residues of Municipal Newsprint Wastes. Forest Products Journal. 47(10): 107 - 110. 